\title{
Robust stabilization of nonlinear systems by optimal controllers
}

\author{
J. Kabziński \\ Institute of Automatic Control, Technical University \\ PL 90-924 Lódź, Stefanowskiego 18/22
}

\begin{abstract}
In this note we investigate robustness of optimally controlled systems in presence of nonlinear state-depending perturbations effecting the input additively together with nonlinear change in the control gain. We derive two types of robustness conditions for optimal controller. The first one can be verified a priori and the second - if the optimal control problem is solved. In the next section we propose robust controller design technique without solving optimal control problem. Next we study special systems and give numerical examples.
\end{abstract}

\begin{abstract}
Keywords
Nonlinear control, robust control, optimal control
\end{abstract}

1 INTRODUCTION

It is well recognized by control scientists that robustness is a basic desirable feature in the design of control systems. It is also known that optimally controlled systems possesses this property.

Let us consider a system described by differential equation:

$\dot{x}(t)-f(x(t), u(t)), \quad f(0,0)=0, \quad x(t) \in R^{n}, \quad u(t) \in R^{m}$.

The control $u(\cdot)$ is chosen to minimize the performance index

$J-\int_{0}^{\infty}(g(x(t))+h(u(t))) d t, \quad g(0)-0, \quad g(x)>0 \quad$ for $x \neq 0, \quad h(0)-0, \quad h(u)>0$ for $u \neq 0$.

We will assume that the data of the above problem are sufficiently smooth to guarantee existence of optimal control in the feedback form $k: R^{n} \rightarrow R^{m}, u(t)=k(x(t))$ and that the optimal cost-to-go value function $V(\cdot)$ is continuously differentiable and satisfies Hamilton-Jacobi-Bellman equation:

$\bigvee x \in R^{n}, u \in R^{m} \quad V_{x}^{T} f(x, u)+g(x)+h(u) \geq V_{x}^{T} f(x, k(x))+g(x)+h(k(x))-0$

Under this optimal control law the origin is globally asymptotically stable equilibrium point of the closed loop system (if we assume $g(x) \geq 0$ instead of $g(x)>0$ for $x \neq 0$ local observability condition is necessary to derive asymptotical stability (Glad, 1984)). 
The optimality of the control implies also that the closed loop system is robust in presence of perturbations in the feedback loop (Tsitsiklis, Athans 1984, Glad 1984, Geromel, Yamakami 1985). Under some additional constraints the closed loop system has a guaranteed infinite gain margin and $50 \%$ gain reduction margin and 60 degree phase margin in each feedback channel (Tsitsiklis, Athans 1984).

In this note we investigate robustness of optimally controlled systems in presence of nonlinear state-depending perturbations effecting the input additively together with nonlinear change in the control gain. we derive two types of robustness conditions for optimal controller. The first one can be verified a priori and the second - if the optimal control problem is solved. In the next section we develop ideas from Kabziński (1991) to propose robust controller design technique without solving optimal control problem. Next we study special systems and give numerical examples.

\section{ROBUSTNESS OF OPTIMAL CONTROLLER}

Let us consider a nominal system being a special form of (1):

$\dot{x}(t)-A(x(t))+B(x(t)) u(t), \quad A(0)=0$

and a special form of a performance index:

$J=\int_{0}^{\infty}\left(g_{0}(x(t))+n(x(t))+\frac{1}{2} u(t)^{T} R u(t)\right) d t, \quad g_{0}(0)-0, \quad g_{0}(x)>0$ for $x \neq 0, \quad n(0)-0$

where matrix $R$ is positive definite. The optimal feedback control law is given by:

$k(x)=-R^{-1} B^{T}(x) V_{x}(x)$.

We investigate robustness of the optimal controller subject to non-linear, state dependant perturbation effecting the input channel. The perturbed system is described by:

$\ddot{x}(t)-A(x(t))+B(x(t))[d(x(t))+p(u(t))]$,

where $d(\cdot)$ and $p(\cdot)$ are memoryless nonlinearities satisfying $d(0)=0, p(0)=0$ and such that equation (6) has a unique solution define for all positive $t$. For $d(\varphi)=0$ and $p(u)=u$ we get the nominal system.

Theorem 1.

If there exist positive number $b$ such that for every $u$ and $x$ :

$2 p(u)^{T} R u-(1+b) u^{T} R u \geq 0$,

$2 b n(x)-d(x)^{T} R d(x) \geq 0$

then the optimal control law for problem $(4,5)$ is robust stabilizing control for system (7).

Proof:

We try cost-to-go value function for problem $(4,5)$ as Lyapunov function candidate for $(7)$. It 
follows from (6) and (3) that the system derivative is given by:

$$
\begin{gathered}
\dot{V}(x)=V_{x}(x)^{T}[A(x)+B(x) d(x)+B(x) p(k(x))]-V_{x}(x)^{T}[A(x)+B(x) k(x)]+V_{x}(x)^{T} B(x)[p(k(x))-k(x)]+ \\
+V_{x}(x)^{T} B(x) d(x)--g_{0}(x)-n(x)-\frac{1}{2} k(x)^{T} R k(x)-p(k(x))^{T} R k(x)+k(x)^{T} R k(x)-k(x)^{T} R d(x)- \\
=-g_{0}(x)-n(x)+\frac{1}{2} k(x)^{T} R k(x)-k(x)^{T} R d(x)-p(k(x))^{T} R k(x) .
\end{gathered}
$$

After adding and subtracting $b k(x)^{T} R k(x)+\frac{1}{b} d(x)^{T} R d(x)$ we are able to rewrite (10) in the following form:

$$
\begin{gathered}
\dot{V}(x)-\frac{1}{2}\left\{-\left[2 k(x)^{T} R p(k(x))-(1+b) k(x)^{T} R k(x)\right]-\frac{1}{b}[d(x)+b k(x)]^{T} R[d(x)+b k(x)]+\right. \\
\left.-\frac{1}{b}\left[2 b n(x)-d(x)^{T} R d(x)\right]\right\}-g_{0}(x),
\end{gathered}
$$

so under $(8,9) \dot{V}(\cdot)$ is negative definite. $\square$

Theorem 1 formulates conditions that do not depend on the optimal control problem solution, allowing then a priori analysis of the closed loop system stability. The derivation of the robust controller requires however the solution of the nonlinear optimal control problem (NOCP).

If $d(x) \equiv 0$, then assumption (8) is satisfied if

$2 p(u)^{T} R u-u^{T} R u \geq 0$,

so (12) generalizes a familiar $50 \%$ gain reduction robustness condition.

If $p(u)=u$ then condition (8) is satisfied with $b=l$ and (9) is reduced to:

$2 n(x)-d(x)^{T} R d(x) \geq 0$,

what is familiar from Geromel and Yamakami (1985). If $n(x)$ is chosen to be a quadratic form $n(x)=1 / 2 x^{T} Q x$ we can replace (9) by a more restrictive condition:

$\frac{\|d(x)\|^{2}}{\|x\|^{2}} \leq \frac{b \lambda_{\min }(Q)}{\lambda_{\max }(R)}$,

where $\lambda_{\text {min }}$ and $\lambda_{\text {max }}$ are minimal and maximal eigenvalue.

Function $n(x)$ is designed to compensate nonlinear state dependent disturbance $d(x)$, while function $g_{d}(x)$ influences the system convergence rate under the worst possible disturbance, what is illustrated by the inequality $\dot{V}(x) \leq-g_{0}(x)$. If $V(x)=x^{T} H x, g_{0}(x) \geq x^{T} G x$, then for any solution of (7) under control (6) $\|x(t)\|^{2} \leq \frac{\lambda_{\max }(H)}{\lambda_{\min }(H)}\|x(0)\|^{2} \exp \left(-\frac{\lambda_{\min }(G)}{\lambda_{\max }(H)} t\right)$.

\section{Theorem 2.}

Assume that $k(x)$ is the optimal control law for problem $(4,5)$. Assume that condition (8) is satisfied and that in some neighborhood $\theta$ of $x=0$, for $x \neq 0$ : 
$2 k(x)^{T} R d(x)+b k(x)^{r} R k(x)+2 n(x)>0$,

then $x=0$ is the asymptotically stable equilibrium point of system (7). The stability domain is given by $\Omega-\left\{x: V(x) \leq \inf _{x \in \partial \theta} V(x)\right\}$.

Proof:

It follows easily from (11) that (15) is sufficient for $\dot{V}(x) \leq-g_{0}(x)$. in $\Theta$.

Conditions $(8,15)$ are less conservative than $(8,9)$, but to verify $(15)$ it is necessary to solve NOCP and obtain $k(x)$.

\section{ROBUST CONTROLLER DESIGN WITHOUT SOLVING OPTIMAL CONTROL PROBLEM}

The derivation of the robust controller proposed in section 2 requires the solution of the nonlinear optimal control problem (NOCP). Solving a NOCP is always a difficult task, although there are several methods, which can be helpful in solving $(4,5)$. Let us investigate if we are able to utilize theorem 1 without solving a NOCP.

Theorem 3.

Assume that condition (8) is satisfied and that there exist positive definite function $V(\cdot)$ and positive numbers $a, b$, such that in a neighborhood $\theta$ of $x=0$, for $x \neq 0$ :

$\left[-V_{x}(x)^{T} A(x)+\frac{1}{2} V_{x}(x)^{T} B(x) R^{-1} B(x)^{T} V_{x}(x)\right] 2 b(1-a)-d(x)^{T} R d(x)>0$,

or:

$a\left[-V_{x}(x)^{T} A(x)+\frac{1}{2} V_{x}(x)^{T} B(x) R^{-1} B(x)^{T} V_{x}(x)\right]>0$,

$-V_{x}(x)^{T} B(x) d(x)-(1-a) V_{x}(x)^{T} A(x)+\frac{1}{2}(1-a+b) V_{x}(x)^{T} B(x) R^{-1} B(x)^{T} V_{x}(x)>0$,

then under the control law: $k(x)=-R^{-1} B^{T}(x) V_{x}(x) \quad x=0$ is the asymptotically stable equilibrium point of system (7). The stability domain is given by $\Omega-\left\{x: V(x) \leq \inf f_{x \in a \theta} V(x)\right\}$.

Proof:

Indeed the control law $k(x)$ is optimal control for system (4) with the performance index (5) where:

$g(x)=g_{0}(x)+n(x)=-V_{x}(x)^{T} A(x)+\frac{1}{2} V_{x}(x)^{T} B(x) R^{-1} B(x)^{T} V_{x}(x), \quad n(x)-(1-a) g(x), \quad g_{0}(x)-a g(x)$.

It follows from (16) that condition (9) is satisfied and the thesis follows like in the proof of theorem 1. Condition (18) is equivalent to inequality (15), condition (17) ensures that $g_{0}(x)>0$ and the proof proceeds like in theorem 2.

The design technique introduced by theorem 3 consists in selecting function $V(\cdot)$. This function is connected with the open loop system and it parameterizes the optimal control and the performance index.

The function $V(x)$ satisfying (16) is easy to find if the system without control $\dot{x}(t)-A(x(t))$ is 
stable. Then there exists Lyapunov function $V(\cdot)$, such that $-V_{x}(x)^{T} A(x)>0$. Parameterization of $V(\cdot)$ allows to satisfy (16) for a given class of disturbances $d(x)$.

In many important cases including bilinear systems $A(x)-A x$. If matrix $A$ is stable then there exist positive definite matrices $P$ and $Q$ satisfying Lyapunov equation:

$P A+A^{T} P=-Q$

and Lyapunov function is $V(x)=\frac{1}{2} x^{T} P x$.. Condition (16) takes the form:

$\left[x^{T} Q x+V_{x}(x)^{T} B(x) R^{-1} B(x)^{T} V_{x}(x)\right] b(1-a)-d(x)^{T} R d(x)>0$,

If matrix $A$ is unstable $Q$ is not positive definite and inequality (21) can be satisfied only locally.

\section{EXAMPLES}

\subsection{Robustness of optimal regulator}

Let us consider a scalar NOCP defined by the nominal system and the performance index:

$\dot{x}=-1.4 x-0.5 x^{2}+(0.5-2.5 x) u, \quad x(0)<0$,

$J-\int_{0}^{\infty}\left(1.2 x^{2}+\frac{1}{2} u^{2}\right) d t$

As it is a scalar problem we are able to solve HJB equation and to find optimal control:

$k(x)=\frac{\left(1.4+0.5 x^{2}\right)-\sqrt{ }\left(x^{2}\left(25.25 x^{2}-8.6 x+2.96\right)\right)}{(1-5 x)}$.

If we take $p(u)=u$ and $g_{o}(x)=\varepsilon x^{2}$ we obtain for $\varepsilon \rightarrow 0 \quad n(x)<1.2 x^{2}$. Sets defined by condition (9) and (15) are plotted in fig. 1 , together with disturbances $d(x)=-0.5 x^{2}, d(x)=1.5 x, d(x)=-1.5 x$. State histories corresponding to these disturbances, under optimal control $k(x)$, starting from $x=-3.05$ are plotted in fig. 2.

\subsection{Robust controller synthesis for nonlinear system}

Let us consider nominal system described by equation (4), where:

$x=\left[\begin{array}{c}x_{1} \\ x_{2}\end{array}\right], \quad A(x)-10\left[\begin{array}{c}-x_{1}^{3}-x_{2} \\ x_{1}-x_{2}^{5}\end{array}\right], \quad B(x)=10\left[\begin{array}{c}2 x_{1} x_{2} \\ x_{2}+1\end{array}\right]$.

We look for Lyapunov function for the system $\dot{x}-A(x)$. We can use Krasovski method and choose $V\left(x_{1}, x_{2}\right)=\frac{1}{2}\left(x_{1}^{3}+x_{2}\right)^{2}+\frac{1}{2}\left(x_{1}-x_{2}^{5}\right)^{2}, \quad V_{x}(x)-\left[\begin{array}{c}3 x_{1}^{2}\left(x_{1}^{3}+x_{2}\right)^{2}+x_{1}-x_{2}^{5} \\ x_{1}^{3}+x_{2}-5 x_{2}^{4}\left(x_{1}-x_{2}^{5}\right)\end{array}\right]$. 
The optimal stabilizing feedback is given by:

$k\left(x_{1}, x_{2}\right)=-\frac{10}{R}\left[\begin{array}{ll}2 x_{1} x_{2} & x_{2}+1\end{array}\right]\left[\begin{array}{c}3 x_{1}^{2}\left(x_{1}^{3}+x_{2}\right)^{2}+x_{1}-x_{2}^{3} \\ x_{1}^{3}+x_{2}-5 x_{2}^{4}\left(x_{1}-x_{2}^{5}\right)\end{array}\right]$.

If we choose $V\left(x_{1}, x_{2}\right)-\frac{1}{2}\left(x_{1}^{2}+x_{2}^{2}\right)$ we obtain respectively:

$V_{x}(x)=\left[\begin{array}{c}x_{1} \\ x_{2}\end{array}\right], \quad k\left(x_{1}, x_{2}\right)=-\frac{10}{R}\left[\begin{array}{ll}2 x_{1} x_{2} & x_{2}+1\end{array}\right]\left[\begin{array}{c}x_{1} \\ x_{2}\end{array}\right]$.

As the input is scalar, condition (16) is reduced to:

$|d(x)|<\left\{\frac{2 b(1-a)}{R}\left[-V_{x}(x)^{T} A(x)+\frac{1}{2} V_{x}(x)^{T} B(x) R^{-1} B(x)^{T} V_{x}(x)\right]\right\}^{1 / 2}$.

The region defined by this condition is plotted for $R=20, b=1, a-0$ in fig. 3 and 4 for two choices of $V(x)$. The corresponding regions defined by (18) are shown in fig. 5 and 6.

\subsection{Global robust stabilizer for bilinear system}

Let us consider bilinear system:

$\dot{x}=A x+B(x) u-A x+\left(B_{0^{+}} B_{1}(x)\right) u, \quad A-\left[\begin{array}{cc}0 & 1 \\ -5 & 2\end{array}\right], \quad B_{0^{-}}\left[\begin{array}{l}1 \\ 0\end{array}\right], \quad B_{1^{-}}\left[\begin{array}{c}2 x_{2} \\ x_{1}\end{array}\right]$.

Linear stabilizing feedback was proposed for this system by Derese and Noldus (1980), but under this control only local stability is possible. Let us consider disturbances $\mathrm{d}(\mathrm{x})$ satisfying:

$d(x)^{2} \leq x^{T} Q_{1} x, \quad Q_{1}=(1-a) Q-0.7\left[\begin{array}{rr}1 & 0 \\ 0 & 10\end{array}\right]$.

Then matrix $P$ is calculated from (20) and the stabilizing control is given by $k(x)=-R^{I} B(x)^{r} P x$. Condition (21) is (conservatively) satisfied with $\mathrm{R}=1, \mathrm{~b}=1$, for $d(x)$ satisfying (31). The system was tested for $d(x)=V 0.7 x_{f}, d(x)=\checkmark 7 x_{2} d(x)=0$. State trajectories are plotted in fig 7. All starting points are chosen outside stability regions obtained by Derese and Noldus (1980).

\section{$5 \quad$ CONCLUSIONS}

We have investigated robustness of optimally controlled nonlinear systems in presence of nonlinear state-depending perturbations effecting the input additively together with nonlinear 


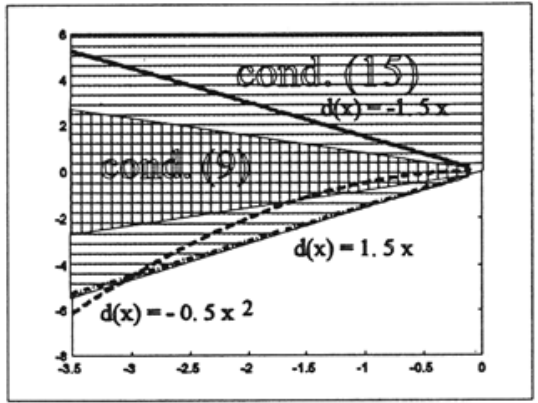

Figure 1 Disturbances and robust stability regions for example 4.1.

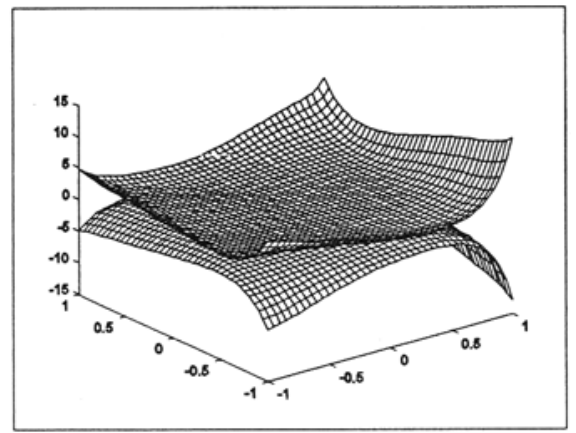

Figure 3 A priori disturbance bounds $(V(x)$ from Krasovski method, example 4.2).

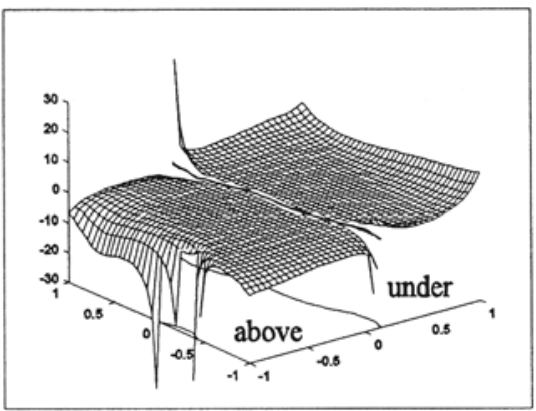

Figure 5 A posteriori robustness regions $(V(x)$ from Krasovski method, example 4.2).

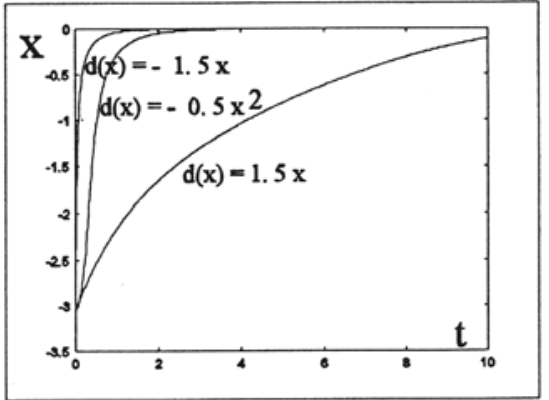

Figure 2 State histories for different d(x) (example 4.1).

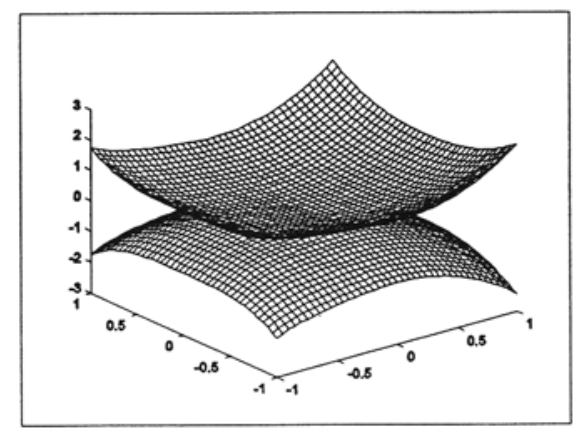

Figure 4 A priori disturbance bounds $(V(x)$ - quadratic form, example 4.2).

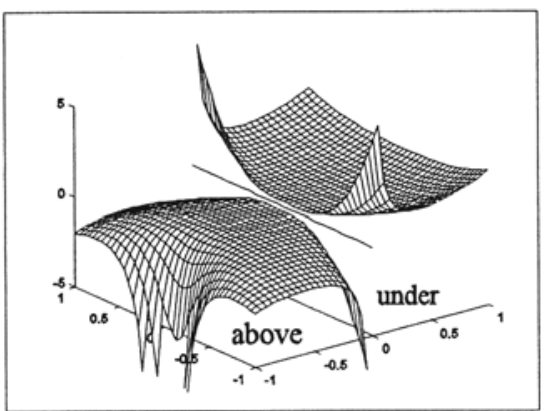

Figure 6 A posteriori robustness regions $(V(x)$ -quadratic form, example 4.2). 
change in the control gain. We derived two types of robustness conditions for optimal controller. The first one can be verified $a$ priori and the second - if the optimal control problem is solved. The first condition is far more conservative as it was shown in example 4.1. The designer selects the performance index to obtain satisfactory

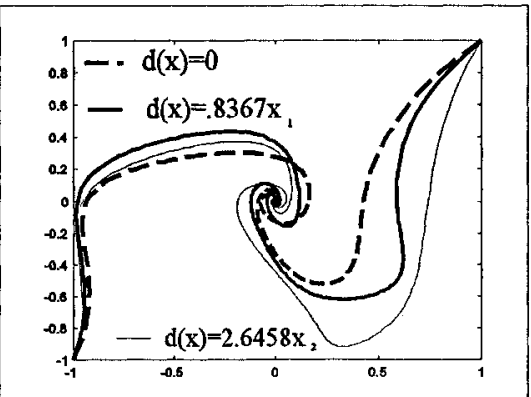

Figure 7 State trajectories under stabilizing

control for several disturbances (example 4.3) robustness bonds for disturbances, good system behavior and bounded control signals. Then nonlinear optimal control problem is solved. If it is too difficult to solve NOCP, we can proceed as in section 3. The designer is to select positive definite function $V(x)$ associated with the system without control and matrix $R$ penalizing controls in the performance index. The stabilizing control is then found without solving optimal control problem. This method is effective for design of robust controllers for nonlinear systems stable without control. Example 4.2 demonstrates how robustness regions are effected by design parameters and example 4.3 shows how to obtain the controller for given a priori disturbance bounds.

\section{REFERENCES}

Derese L. and Noldus I., (1980) Design of linear feedback laws for bilinear systems, Int. Jour. Control, vol. 31, 219-237

Geromel, J.C. and Yamakami A.. (1985) On the robustness of nonlinear regulators and its application to nonlinear systems stabilization, IEEE Trans. Automatic Control, vol. AC-30, 1251-1254.

Glad S.T. (1984) On the gain margin of nonlinear and optimal regulators, IEEE Trans. Automatic Control, vol. AC-29, 615-620.

Kabziński J. (1991) Optimal control for stabilization of nonlinear systems, in System Modelling and Optimization, Kall p. ed., Lecture Notes in Control and Inf. Sc., vol 180, 520-529.

Tsitsiklis J.N. and Athans M. (1984) Guaranteed robustness properties of multivariable nonlinear stochastic optimal regulators, IEEE Trans. Automatic Control, vol. AC-29, 690-696. 